\title{
Long-Term Complications of Epidural Steroid Injections
}

\author{
Jae H. Jung • Mark I. Ignatius • Jason M. Davis • \\ Lysander Jim
}

Published online: 16 January 2014

(c) Springer Science + Business Media New York 2014

\begin{abstract}
Epidural steroid injections (ESI) are common pain-relief procedures performed throughout the world, yet there remains controversy regarding their long-term safety. On the basis of current literature, serious long-term complications are rare yet can have devastating results. Potential etiologies for long-term complications associated with ESI include infection, bleeding, endocrine effects, neurotoxicity, and neurologic injury. Despite the multitude of literature on ESI, there is still a lack of studies specifically addressing possible long-term complications. Further research is therefore warranted.
\end{abstract}

Keywords Epidural steroid injection · Long-term complications · Neurologic injury · Neurotoxicity · Arachnoiditis $\cdot$ Spinal hematoma

J. H. Jung $(\bowtie) \cdot$ M. I. Ignatius

Department of Orthopaedics, Physical Medicine and

Rehabilitation, David Geffen School of Medicine,

The UCLA Spine Center, 1250 16th Street, Suite 3145H,

Santa Monica, CA 90404, USA

e-mail: jjung@mednet.ucla.edu

M. I. Ignatius

e-mail: mignatius@mednet.ucla.edu

J. M. Davis · L. Jim

Physical Medicine and Rehabilitation (W117), UCLA/VA

Greater Los Angeles Healthcare System, 11301 Wilshire Blvd., Los Angeles, CA 90073, USA

e-mail: patchatoms@gmail.com

L. Jim

e-mail: lysanderjim@gmail.com

\section{Introduction}

Epidural steroid injections (ESI) are among the most commonly performed pain-relief procedures in modern medicine. Although the literature on the efficacy and safety of ESI is substantial, there remains a paucity of studies addressing possible long-term complications. This may be related to the lack of mandatory reporting of complications from ESI in the US, which also makes determining the true incidence of serious adverse effects essentially impossible.

There was much debate about the overall safety of ESI with the unfortunate outbreak of fungal meningitis in the US in 2012, which occurred after administration of contaminated steroid and resulted in more than 700 cases of fungal infection and over 48 deaths [1]. The American Society of Anesthesiologists Closed Claims Study also reported 114 major complications supposedly caused by ESI resulting in malpractice litigation; these included nerve injury, infection, death, brain damage, headache, and increased pain or lack of relief [2].

Despite controversy about safety, current literature suggests serious long-term complications as a result of epidural steroid administration are rare. A recent retrospective analysis studied 1,857 patients who received, over a seven-year period, 4,265 ESI, including 123 lumbar interlaminar injections, 17 caudal injections, 3,964 lumbar transforaminal injections, and 161 cervical interlaminar injections. The study identified an incidence of complications of $2.4 \%$ per injection [3•]. The complications were all determined to be minor, consisting of increased pain, pain at the injection site, persistent numbness, or "other", with no major complications reported. Another study, from 2000, of 270 patients who received 322 fluoroscopically guided transforaminal epidural injections found the incidence of minor complications to be $9.6 \%$ per injection; there were no major complications [4]. 
Despite the rarity of serious adverse effects after ESI, the long-term complications that have been reported arise from both mechanical and chemical sources. These potential long-term complications are primarily related to infection, bleeding, endocrine effects, and neurologic complications.

\section{Infection}

Infectious complications after ESI are rare. They include such significant infections as epidural abscess, discitis, osteomyelitis, and meningitis. Among the conditions listed, the highest incidence is of spinal epidural abscess, but this accounts for only 1-2 cases per 10,000 among all patients admitted to hospital [2]. Although back pain, fever, and leukocytosis are the classical features, epidural abscess may mimic other medical conditions. The patient may present with abdominal or flank pain in the absence of fever, neck stiffness, or elevated white cell count [5].

Tang et al. [6] characterized the prognostic features of spinal epidural abscess by retrospective chart review of 46 patients. It was found that a high proportion of patients had underlying diabetes $(46 \%)$, frequent venous puncture (35\%), spinal trauma (24\%), and history of spinal surgery $(22 \%)$. Localized spinal pain $(89 \%)$, paralysis $(80 \%)$, fever and/or chills $(67 \%)$, and radicular pain $(57 \%)$ were the most common manifestations. The erythrocyte sedimentation rate (ESR) was elevated in all patients for whom this was measured, suggesting ESR enables highly sensitive study for epidural abscess. The efficacy of appropriate treatment in restoring neurological function has been documented by case reports of patients who developed spinal epidural abscess after ESI [7].

Widespread outbreaks of infection after ESI are nearly always associated with contaminated injectate. At the time of writing, the most recent instance was a widespread outbreak of fungal infections in 2012, because of contaminated methylprednisolone acetate prepared by a single compounding pharmacy [8]. Aspergillus fumigatus and Exserohilum rostratum contamination of vials of methylprednisolone were associated with meningitis, cauda equina syndrome, focal infection, and posterior circulation stroke. According to the Center for Disease Control, more than 48 deaths and 700 instances of illness were associated with this outbreak. Clinical and regulatory oversight are both required to ensure the sterile conditions necessary to limit infections from ESI.

\section{Bleeding}

Bleeding occurs with a range of clinical severity from injection site bruising to neurologically catastrophic epidural hematoma. The prevalence of all bleeding complications is low and one prospective evaluation of 10,000 fluoroscopically directed epidural injections observed the intravascular entry of needle in $4.3 \%$ of cases, return of blood on aspiration in $1.5 \%$, profuse bleeding in $0.5 \%$, local hematoma in $0.1 \%$, bruising in $0.2 \%$, and no epidural hematomas [9•].

Although rare, an epidural hematoma is a potentially devastating complication of epidural steroid injection. The reported incidence of spinal hematoma after intervention is estimated to be between 1 in 70,000 and 1 in 190,000 [10, 11]. A case report notes some classical features of this complication for a patient who had received a cervical ESI: severe pain around the injection site that evolved within hours to urinary symptoms, ataxia, and flaccid paralysis of the bilateral lower extremity [12]. A spinal epidural hematoma occurs when buildup of blood occurs between the dura mater and the spinal cord, often because of iatrogenic trauma of an epidural vein. Hematogenous compression of the spinal cord can lead to permanent paralysis and death if not treated as an emergency by surgical evacuation.

Suspension of antithrombotic and thrombolytic therapy before an epidural steroid injection is common practice [13]. An estimated $25 \%$ of patients presenting for interventional pain management techniques are on antithrombotic therapy $[14,15]$. Although recommendations have been made by a variety of societies, including the Third Consensus Conference on Regional Anesthesia and Anticoagulation, there is a limitation on the use of evidence based reviews because the rarity of spinal hematoma defies a prospective randomized study, and there is no current laboratory model. According to Horlocker et al. [15] there were no reported major hemorrhagic complications among 417 patients who underwent ESI and were taking aspirin, NSAIDs, or multiple drugs. Although there was no appreciable increase in risk of bleeding, the rarity of spinal hematoma precludes more definitive assessment of risk. Manchikanti et al. have noted that the risk of bleeding during interventional pain management for patients undergoing antiplatelet therapy is scant to nonexistent $[16$, 17]. The authors note that their survey of interventional pain practitioners found the risk of complications from thromboembolism was three times more prevalent than epidural hematomas, and concluded that clinicians must balance the risk of thromboembolism and bleeding for each patient before routine discontinuation of antiplatelet therapy. In contradiction of this recommendation, the same survey found that in actual practice, $100 \%$ of physicians discontinued warfarin, $97 \%$ discontinued clopidogrel, $96 \%$ discontinued ticlodipine, $95 \%$ discontinued tirofiban, $93 \%$ discontinued cilostazol, $85 \%$ discontinued dipyridamole, $60 \%$ discontinued aspirin $350 \mathrm{mg}, 39 \%$ 
discontinued aspirin $81 \mathrm{mg}$, and $39 \%$ discontinued nonsteroidal anti-inflammatory drugs before performing interventional pain management. An INR of 1.5 or less was regarded as safe. Thus shortage of quality evidence and the bias toward preventing epidural hematomas over prevention of thrombosis has led to a focus that generally errs toward the side of antiplatelet and anticoagulant cessation before ESI.

\section{Endocrine Complications}

The effect of epidural steroids on the hypothalamic-pituitary-adrenal (HPA) axis was first noted in case reports [1820]. Knight and Burnell [18] documented four case histories of patients who developed cushingoid symptoms after injections of methylprednisolone and bupivacaine into the epidural space. The symptoms emerged about a month after the intervention and included malaise, facial swelling, palpitations, vocal weakness, small raised scaly lesions on the scalp and over the upper trunk, and a hump between the shoulder blades. Of note, the patients received high doses of methylprednisolone ranging from a total of 160 to $600 \mathrm{mg}$ over a three-day period.

In another case report, diagnosis of an exogenous hypercortisolism was confirmed by laboratory studies [19]. The subject was an elderly woman who had received a total of $160 \mathrm{mg}$ over a two-week period and was admitted to the hospital for weight gain and fluid retention. The patient had a reduced morning and afternoon cortisol level on the day after admission of 3.5 and 2.2 micrograms, respectively. A week after admission the patient had an increased cortisol level in an ACTH stimulation test. By four weeks after admission the patient had normal morning cortisol levels and by six weeks after admission had resolution of clinical stigmata of her cushingoid syndrome.

Kay et al. [21] prospectively studied the effect on the endocrine system of epidural steroids with and without midazolam. Midazolam, similar to other benzodiazepines, suppresses the HPA axis. The study involved injecting $80 \mathrm{mg}$ triamcinolone acetate in three consecutive weeks with measurements of $\mathrm{ACTH}$ and cortisol response to cosyntropin. The study demonstrated acute suppression of both plasma ACTH and cortisol after an $80-\mathrm{mg}$ dose of epidural triamcinolone: "The suppression of ACTH started early (within $15 \mathrm{~min}$ ) in the midazolam-premedicated subjects and preceded the decrease in cortisol. Both groups showed reduction of plasma ACTH and cortisol by $45 \mathrm{~min}$ after the first ESI." Furthermore, the study found that 7, 14, and 48 days after the first epidural the subjects who had received midazolam had more highly suppressed ACTH and cortisol levels. After 48 days, only the group that had received midazolam before each ESI had significantly suppressed levels; the group that had not received midazolam had normalized levels. Normalization of the HPA axis for all subjects occurred by 3 months. This study demonstrates that serologically detectable suppression of the HPA axis occurs within an hour after a single clinically used dose, is amplified by concomitant administration of midazolam, and would be expected to return to baseline after cessation of exogenous steroids. Although uncommon, the development of Cushing's syndrome and steroid myopathy has been observed after a single epidural injection of $60 \mathrm{mg}$ triamcinolone acetate [22].

In contrast with the effect on the HPA axis, epidural steroid injections have a clinically limited effect on blood glucose levels among patients with diabetes mellitus. One study found that blood glucose levels after an ESI rose from a baseline value of $160.18 \pm 47.46 \mathrm{mg} / \mathrm{dl}$ to an average of $286.13 \pm 111.11 \mathrm{mg} / \mathrm{dl}$ [23]. The average increase of $125.96 \mathrm{mg} / \mathrm{dl}$ persisted for an average of 1.06 days with a subsequent return to baseline blood glucose levels within two days. There was no association between the degree of change in blood sugar level and the hemoglobin A1C level. Another prospective study found there were no significant changes, as measured by a continuous blood glucose measurement device one day before and two days after injection, among five diabetic patients who had received $80 \mathrm{mg}$ methylprednisolone acetate [24]. These findings suggest that epidural injection of standard doses of steroids does not adversely affect glycemic control in a clinically relevant manner, and any persistent elevation of blood glucose beyond baseline levels should prompt a search for an alternative etiology, for example infection.

\section{Neurologic Complications-Neurologic Injury and Neurotoxicity}

Similar to the complications listed above, neurotoxicity and neurologic injury are rare and, given the lack of mandatory reporting, as already mentioned, data on the incidence and prevalence of long-term complications from ESI are lacking. Case reports have shown that when neurologic complications do occur they can have devastating effects that lead to long-term and even permanent neurologic deficits and pain. The most common causes of neurologic injury seem to be direct needle trauma to the spinal cord or nerve roots, intra-vascular injection of steroid leading to infarction, and compression of the spinal cord and roots related to an epidural hematoma or abscess [25, 29]. Less commonly, direct compression of the surrounding neurologic structures, for example the dorsal root ganglia, can occur [26]. Indirect compression or injury of the spinal cord and/or nerve roots related to infectious or inflammatory processes, for example abscesses, arachnoiditis, and 
meningitis, as mentioned above, can also cause neurologic injury. These complications can often be avoided by appropriate patient selection and use of suitable techniques [26].

The possibility of long-term neurologic deficits should be considered for any patient with significant new neurologic deficits in the acute and subacute post-procedure period. Acute examples include immediate hemiplegia, paraplegia, or quadriplegia after possible direct spinal cord needle trauma or intravascular steroid injection. Subacute examples include progressive weakness a few days to weeks post procedure related to an epidural abscess or hematoma causing spinal cord compromise. New or progressive neurologic complications several months or years after ESI seem to be exceedingly rare but have been documented in atypical cases of delayed occurrence of arachnoiditis [27, 28].

The American Society of Anesthesiologists Closed Claims Study published in 2004 found and evaluated 114 claims from 35 professional liability companies between 1970 and 1999 related to epidural injections with or without local anesthetic [2]. Of the 114 claims, nine (8\%) were related to death or brain damage, $28(25 \%)$ were related to nerve injury, $24(21 \%)$ were related to infection, and $20(18 \%)$ were related to headache. Among the 28 nerve injury claims, seven (6\% of the 114 ESI claims) resulted in severe spinal cord injury (six paraplegia and one quadriplegia).

In 2011, a follow-up study [29] by the Closed Claims Group specifically evaluating malpractice claims related to interventional pain treatment of the cervical spine between 2005 and the end of 2008 was published. During this time period, there were 43 claims specifically related to cervical epidural injections (95\% used steroids). This study reported combined damaging outcome results of all cervical procedure cases and not just epidural injections. This included a total of 64 claims, most of which were procedural: 43 epidural injections, seven stellate ganglion blocks, six trigger point injections, and two intra-articular facet injections. Twenty percent of the claims were nonprocedure-related, for example failure to diagnose, patient fall, wrong procedure, etc. Of the procedurally based claims (51 out of 64), direct cord or nerve trauma from the needle was the most common claim (20 claims out of 51 or $39 \%$ of procedural claims) and spinal cord infarction and/ or stroke after intra-arterial injection was the second most common (nine claims or $18 \%$ of procedural claims). This was followed by four claims of dural puncture and three claims each of hematoma causing spinal cord compression, infection and/or abscess, and high total spinal block.

A sub-analysis was performed of the 38 patients with spinal cord injury claims. Direct needle trauma was determined to be the most common cause of spinal cord injury (SCI) with 20 claims, of which six SCI claims were related to cord infarction from an intra-arterial injection and three SCI claims were related to cord compression from a hematoma. Of the SCI patients, $33(87 \%)$ had permanent disabling injuries that resulted in nine patients with quadriplegia-quadraparesis, six with paraplegia and/ or paraparesis, and three with hemiplegia and/or hemiparesis [29].

Arachnoiditis, more severe chronic adhesive arachnoiditis, and aseptic meningitis are other feared serious complications that could potentially lead to significant long-term complications. The incidence and prevalence of each after epidural steroid injections are unclear, but these also seem to be quite rare. A direct neurotoxic effect of corticosteroid suspensions has often been suggested, although not definitively proved for humans [30••]. Arachnoiditis, or inflammation of the meninges and subarachnoid space, can be caused by a multitude of other etiologies, including infection, previous surgery, dural tears, bleeding, myelograms, and intrathecal anesthesia [31]. If this inflammation persists, scarring, fibrosis, and adhesion can lead to chronic pain syndromes and neurologic deficits (weakness, numbness, cauda equina syndrome) some of which can be progressive. Aseptic meningitis, which is caused by serous inflammation of the meninges, often presents with a fever, headache, and altered mental status. It is typically diagnosed after CSF examination revealing a lymphocytic pleocytosis, slightly elevated protein, and normal glucose. The greatest incidence of arachnoiditis and aseptic meningitis seems to occur during intentional or unintentional intrathecal steroid injections and not as a result of epidural steroid injections $[30 \bullet \cdot$. The offending agent within steroid suspensions is unclear. Largely on the basis of bench-top and animal trials, poly(ethylene glycol) and benzyl alcohol have been most suspected of causing neurotoxic effects. Celestone, a betamethasone product in an aqueous vehicle without those agents, is believed to be possibly less neurotoxic and potentially less likely to lead to arachnoiditis. It should be noted, however, that an intrathecal study of Celestone revealed histologic evidence of arachnoiditis [30••], and it is likely some risk is associated with most commercial steroid preparations.

Most SCI and nerve injuries seem to occur when a patient is over-sedated and fails to respond appropriately to direct needle compression or injection. Infarctions and strokes tend to occur most frequently after cervical ESI with use of large particulate suspensions. Arachnoiditis and aseptic meningitis possibly related to a neurotoxic effect of the steroid suspension are, by far, most common with intrathecal injections and not epidural injections. These complications can largely be avoided with simple precautions and appropriate technique, for example avoiding 
over-sedation, use of a more soluble steroid, for example dexamethasone, and use of a radiocontrast dye and/or local anesthetic test dose to prevent an unintentional intrathecal injection $[25,30 \bullet \cdot$.

\section{Conclusion}

Long-term complications from ESI are rare overall, but can be devastating, especially when involving a serious neurologic injury. Most long-term complications arise from acute injuries that worsen over time rather than from longterm effects that occur directly from the steroid injection itself. Much research has been conducted to address the efficacy and safety of ESI, although little research has focused specifically on long-term complications. This could be related to under-reporting, because there is currently no requirement in the US to report complications from ESI when they do occur. Nevertheless, the current literature shows that serious long-term complications from ESI are uncommon, but given the lack of literature specifically directed at potential long-term complications further investigation with prospective studies is warranted.

\section{Compliance with Ethics Guidelines}

Conflict of Interest J. H. Jung declares no conflicts of interest. M. I. Ignatius declares no conflicts of interest. J. M. Davis declares no conflicts of interest. L. Jim declares no conflicts of interest.

Human and Animal Rights and Informed Consent This article does not contain any studies with human or animal subjects performed by any of the authors.

\section{References}

Papers of particular interest, published recently, have been highlighted as:

- Of importance

•- Of major importance

1. Centers for disease control and prevention, multistate fungal meningitis outbreak investigation. Available at: http://www.cdc. gov/hai/outbreaks/meningitis.html. Accessed 12 Sep 2013.

2. Fitzgibbon DR, Posner KL, Domino KB, Caplan RA, Lee LA, Cheny FW. Chronic pain management: American Society of Anesthesiologists Closed Claims Project. Anesthesiology. 2004;100:98-105.

3. - McGrath J, Schaefer M, Malkamaki D. Incidence and characteristics of complications from epidural steroid injections. Pain Med. 2011;12:726Y731. This publication was a recent detailed review on ESI complication incidence broken up by each injection type.

4. Botwin KP, Gruber RD, Bouchlas CG, Torres-Ramos FM, Sanelli JT, Freeman ED, Slaten WK, Rao S. Fluoroscopically guided lumbar transforaminal epidural steroid injections in degenerative lumbar stenosis: an outcome study. Am J Phys Med Rehabil. 2002;81:898-905.

5. Nussbaum ES, Rigamonti D, Standiford H, Numaguchi Y, Wolf AL, Robinson WL. Spinal epidural abscess: a report of 40 cases and review. Surg Neurol. 1992;38:225-31.

6. Tang H, Lin H, Liu Y, et al. Spinal epidural abscess-experience with 46 patients and evaluation of prognostic factors. J Infect. 2002;45:76Y81.

7. Huang R, Shapiro G, Lim M, et al. Cervical epidural abscess after epidural steroid injection. Spine. 2004;29:E7YE9.

8. Kainer M, Reagan D, Nguyen D, et al. Fungal infections associated with contaminated methylprednisolone in Tennessee. N Engl J Med. 2012;367:2194-203.

9. - Manchikanti L, Malia Y, Wargo B, et al. A prospective evaluation of complications of 10,000 fluoroscopically directed epidural injections. Pain Physician. 2012;15:131-140. This was a prospective look at complications of ESI investigating a particularly high number of ESI.

10. Abram SE, O'Connor TC. Complications associated with epidural steroid injections. Reg Anesth. 1996;21(2):149-62.

11. Wulf H. Epidural anesthesia and spinal hematoma. Can J Anesth. 1996;43(12):1260-71.

12. Williams K, Jackowski A, Evans P. Epidural hematoma requiring surgical decompression following repeated epidural steroid injections for chronic pain. Pain. 1990;42:197Y199.

13. Manchikanti L, Benyamin R, Swicegood J, et al. Assessment of practice patterns of perioperative management of antiplatelet and anticoagulant therapy in interventional pain management. Pain Physician. 2012;15:E955YE968.

14. Manchikanti L, Malla Y, Wargo BW, Cash KA, McManus CD, Damron KS, Jackson SD, Pampati V, Fellows B. A prospective evaluation of bleeding risk of interventional techniques in chronic pain. Pain Physician. 2011;14:317-29.

15. Horlocker TT, Bajwa ZH, Ashraf Z, Khan S, Wilson JL, Sami N, Peeters-Asdourian C, Powers CA, Schroeder DR, Decker PA, Warfield CA. Risk assessment of hemorrhagic complications associated with nonsteroidal anti-inflammatory medications in ambulatory pain clinic patients undergoing epidural steroid injection. Anesth Analg. 2002;95:1691-7.

16. Horlocker TT, Wedel DJ, Schroeder DR, Rose SH, Elliott BA, McGregor DG, Wong GY. Preoperative antiplatelet therapy does not increase the risk of spinal hematoma associated with regional anesthesia. Anesth Analg. 1995;80:303-9.

17. Horlocker TT, Wedel DJ, Offord KP. Does preoperative antiplatelet therapy increase the risk of hemorrhagic complications associated with regional anesthesia? Anesth Analg. 1990;70: $631-4$.

18. Knight C, Burnell J. Systemic side effects of extradural steroids. Anaesthesia. 1980;35:593Y594.

19. Stambough J, Booth R, Rothman R. Transient hypercorticism after epidural steroid injection. J Bone Joint Surg. 1984;66A: $1115 Y 1116$.

20. Tuel S, Meythaler J, Cross L. Cushing's syndrome from methylprednisolone. Pain. 1990;40:81Y84.

21. Kay J, Findling J, Raff H. Epidural triamcinolone suppresses the pituitary-adrenal axis in human subjects. Anesth Analg. 1994;79:501Y505.

22. Boonen S, Van Distel G, Westhovens R, et al. Steroid myopathy induced by epidural triamcinolone injection. $\mathrm{Br} \mathrm{J}$ Rheumatol. 1995;34:385Y386.

23. Even J, Crosby C, Song Y, et al. Effects of epidural steroid injections on blood glucose levels in patients with diabetes mellitus. Spine. 2012;37:E46YE50.

24. Zufferey P, Bulliard C, Gremion G, et al. Systemic effects of epidural methylprednisolone injection on glucose tolerance in diabetic patients. BMC Res Notes. 2011;4:552. 
25. Windsor RE, Storm S, Sugar R. Prevention and management of complications resulting from common spinal injections. Pain Physician. 2003;6:473-83.

26. Goodman BS, Posecion LW, Mallempati S, Bayazitoglu M. Complications and pitfalls of lumbar interlaminar and transforaminal epidural injections. Curr Rev Musculoskelet Med. 2008;1:212-22.

27. Guyer DW, Wiltse LL, Eskay ML, Guyer BH. The long-range prognosis of arachnoiditis. Spine. 1989;14(12):1332-41.

28. Na EH, Han SJ, Kim MH. Delayed occurrence of spinal arachnoiditis following a caudal block. J Spinal Cord Med. 2011;34(6): 616-9.
29. Rathmell JP, Michna E, Fitzgibbon DR, Stephens LS, Posner KL, Domino KB. Injury and liability associated with cervical procedures for chronic pain. Anesthesiology. 2011;114(4):918-26.

30. • Cohen SP, Bicket MC, Jamison D, Wilkinson I, Rathmell JP. Epidural steroids: a comprehensive, evidence-based review. Reg Anesth Pain Med. 2013;38:175-200. This was a very recent ad effective comprehensive overview of ESI which touched on all aspects of ESI including efficacy and complications.

31. Aldrete JA. Chronic adhesive arachnoiditis. $\mathrm{Br} \mathrm{J}$ Anaesth. 2004;93:301-7. 